\title{
Optimizing cerebrovascular surgical and endovascular procedures in children via personalized 3D printing
}

\author{
Peter Weinstock, MD, PhD, ${ }^{1,2,5}$ Sanjay P. Prabhu, MBBS, FRCR, ${ }^{1,3,5}$ Katie Flynn, BS, ME, ${ }^{1}$ \\ Darren B. Orbach, MD, PhD, ${ }^{3,5}$ and Edward Smith, MD ${ }^{4,5}$
}

\begin{abstract}
'Boston Children's Hospital Simulator Program (SIMPeds), ${ }^{2}$ Department of Anesthesiology, Perioperative and Pain Medicine, Division of Critical Care Medicine, and Departments of ${ }^{3}$ Radiology and ${ }^{4}$ Neurosurgery, Boston Children's Hospital and ${ }^{5}$ Harvard Medical School, Boston, Massachusetts
\end{abstract}

\begin{abstract}
OBJECT Despite the availability of multiplanar imaging, understanding relational 3D anatomy for complex cerebrovascular lesions can be difficult. A 3D printed model allows for instantaneous visualization of lesional anatomy from all perspectives, with the added ability to simulate operative approaches with tactile feedback. The authors report their experience with customized 3D printed models of pediatric cerebrovascular lesions as an educational and clinical tool for patients, trainees, and physicians.
\end{abstract}

METHODS Via an "in-house" 3D print service, magnetic resonance imaging (MRI) and computerized tomography (CT) studies of pediatric patients with arteriovenous malformations (AVMs) were processed with specialized software, and regions of interest were selected by the surgical/endovascular team. Multiple models for each patient were then printed on a 3D printer, with each construct designed to illustrate different aspects of the specific lesion. Intraoperative validation of model fidelity was performed using perioperative imaging, surgical filming, and post hoc analysis of models with intraoperative photography.

RESULTS Four cases involving pediatric patients (ages $0-16$ years) were studied for initial proof of principle. Three of the patients had AVMs and one had a vein of Galen malformation (VOGM). The VOGM was embolized successfully and the AVMs were resected without complication. In the AVM cases, intraprocedural imaging and photography were performed and verified millimeter-level fidelity of the models $(n=5,98 \%$ concordance, range $94 \%-100 \%$ with average of < $2 \mathrm{~mm}$ variation in the largest AVM [6-cm diameter]). The use of 3D models was associated with a 30-minute reduction in operative time (12\%) in 2 cases when they were compared with matched controls as a feasibility study.

CONCLUSIONS Patient-specific 3D printed models of pediatric cerebrovascular conditions can be constructed with high fidelity. This proof-of-principle series demonstrates, for the first time, confirmation of model accuracy using intraprocedural assessment and potential benefit through shortened operative time.

http://thejns.org/doi/abs/10.3171/2015.3.PEDS14677

KEY WORDS 3D printing; model; surgery; simulation; pediatric; cerebrovascular; arteriovenous malformation; vein of Galen malformation; technique

$\mathrm{T}$ He recent development of 3D printers has facilitated the creation of models of anatomical structures. This new technology has spurred innovation in several surgical specialties, with isolated reports of using 3D models in orthopedics, urology, and thoracic and plastic surgery. $2,3,6,8,10,11$ These experiences have highlighted the potential of employing $3 \mathrm{D}$ printing in clinical practice as an adjunct for improved understanding of anatomy, for pa- tient communication, and for teaching trainees. To date, there have not been any reports of using this technique in the context of pediatric cerebrovascular disease. Given the uniquely complex anatomy of lesions such as brain arteriovenous malformations (AVMs) and vein of Galen malformations (VOGMs), we describe our initial experience with printing patient-specific 3D models of these conditions with the novel approach of printing "in situ" lesions.

ABBREVIATIONS AVM = arteriovenous malformation; SIMPeds = Boston Children's Hospital Simulator Program; VOGM = vein of Galen malformation . SUBMITTED December 3, 2014. ACCEPTED March 23, 2015.

INCLUDE WHEN CITING Published online July 31, 2015; DOI: 10.3171/2015.3.PEDS14677.

DISCLOSURE This study was supported by the Lucas Warner AVM Research Fund and the Kids at Heart Neurosurgery Research Fund. 
While this is a proof-of-principle study, it provides feasibility data, demonstrating, for the first time, intraoperative validation of model fidelity and evidence that simulation with 3D models may have the potential to reduce operative time.

\section{Methods}

This study was approved by the institutional review board of Boston Children's Hospital.

Four intracranial vascular malformations (3 AVMs and 1 VOGM) were identified as candidates for 3D printing. Selection for printing was based solely on sequential cases in a clinical series due to a planned proof-of-principle collaboration with the Boston Children's Hospital Simulator Program (SIMPeds). The SIMPeds 3D Print Team includes expertise in simulation, mechanical/industrial engineering, radiology, and 3D image processing. Models were constructed based on MRI using selected MR arteriography and post-contrast volumetric sequences. Regions of interest were identified by the treating clinicians and reviewed with the SIMPeds neuroradiologist. The initial segmentation was reviewed and refined to ensure that the pathological vasculature was segmented, and after final review to ensure accuracy of the segmentation, the pathological vasculature was printed along with feeding and draining vessels (Fig. 1). In surgical cases, surrounding brain and all tissue within the planned operative field were also printed. Of note, surgical cases had multiple prints performed, including constructs solely of skeletonized vessels and other constructs including surrounding normal tissues (brain, ventricle, etc.), to visualize both the isolated lesion and the lesion in situ mimicking the surgical approach (Fig. 2).

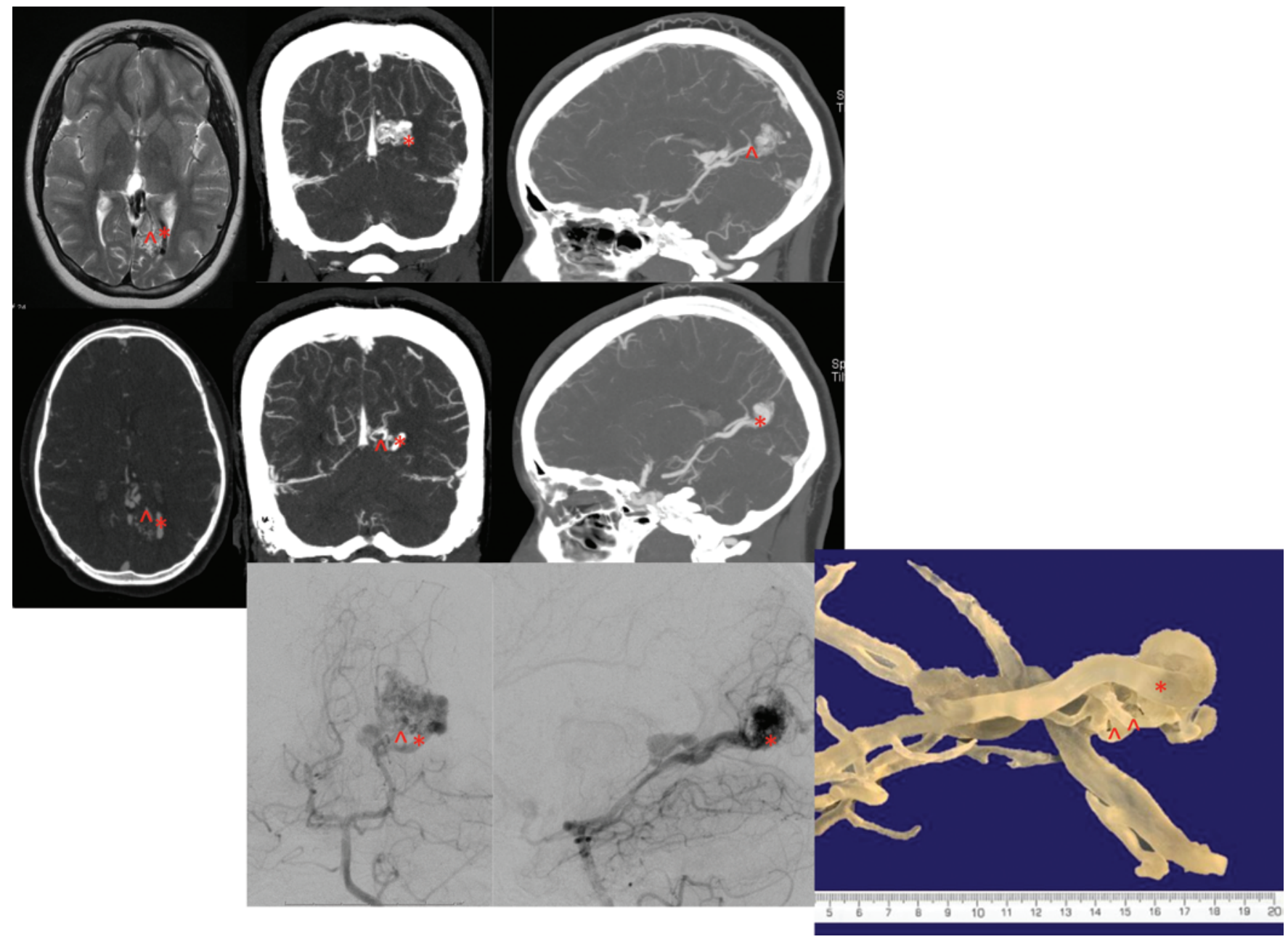

FIG. 1. Despite the use of multiplanar imaging with 3 modalities (MRI, CT angiography, and catheter arteriography), understanding relational 3D anatomy can be difficult. Visualization of detailed anatomical structures can require scrolling through multiple images, and even simulated 3D reconstructions on-screen lack easy manipulation and do not provide tactile feedback. A 3D printed model allows for instantaneous visualization of lesional anatomy from all perspectives, with the added ability to simulate surgical approaches with tactile feedback and use of actual surgical instruments to practice approaches through multiple corridors of attack. Starting in the upper left, clockwise review of 6 images shows an axial T2-weighted MR image, coronal CT angiogram, sagittal CT angiogram, another image of the sagittal CT angiogram, another image of the coronal CT angiogram, and an axial CT angiogram image. Below these are 2 digital subtraction angiograms showing anteroposterior (left) and lateral (right) injections of the vertebrobasilar vessels. (Asterisks denote deep draining vein, carets identify feeding artery.) Note that the model helps to visualize additional feeders, which are more easily seen after printing. 

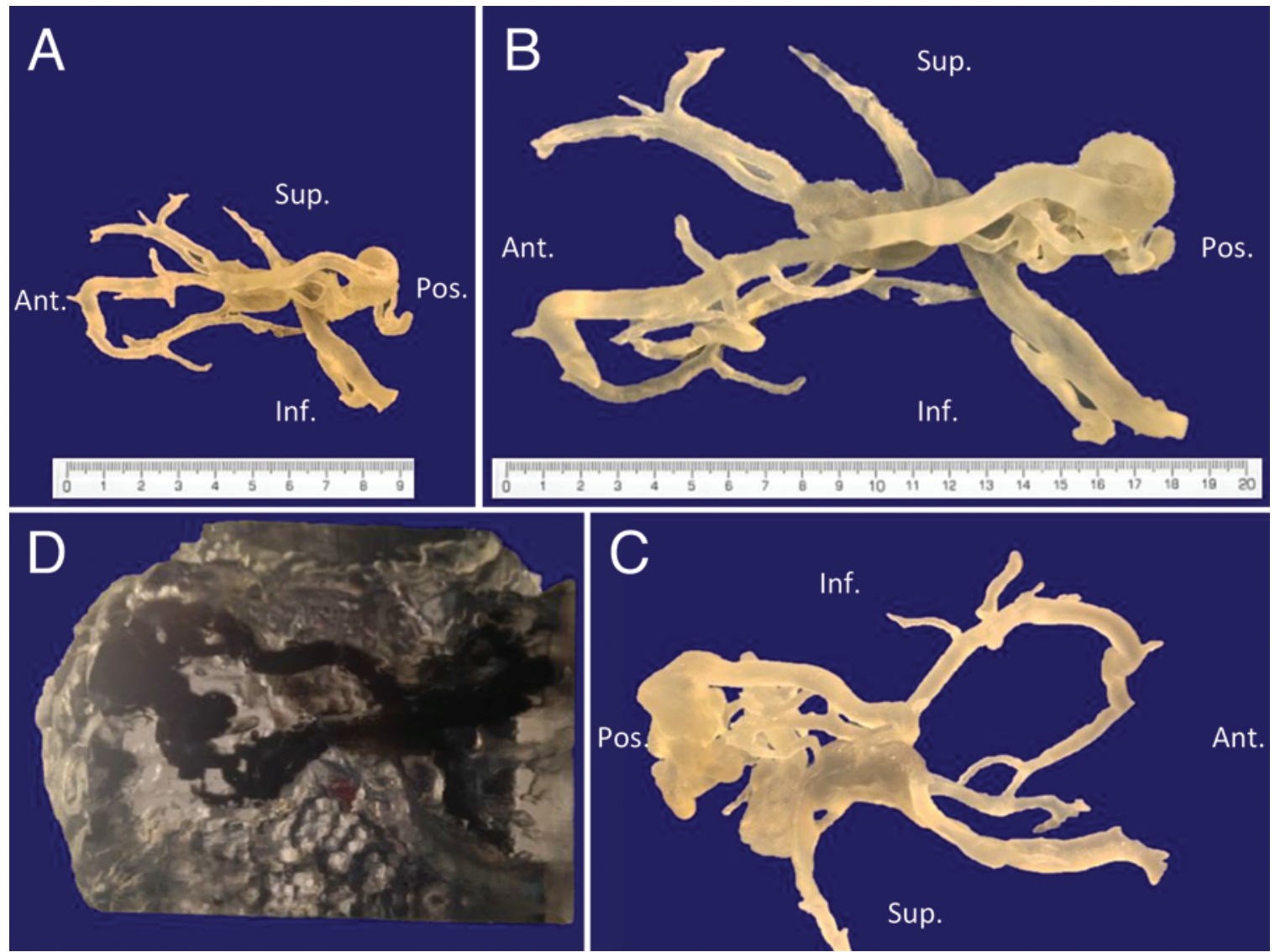

FIG. 2. A-C: Photographs of life-size and enlarged printed models of AVM, viewed from different angles. Note that the model helps to visualize additional feeders, which are more easily seen after printing. Ant. = anterior, Inf. $=$ inferior, Pos. $=$ posterior, Sup. = superior. D: Example of in situ printing of an AVM within clear resin, allowing visualization of the lesion from the surgical viewpoint. This is an occipital lobe AVM, with the nidus and vessels printed in black and the normal occipital lobe in clear resin, enabling the surgeon to view the subcortical lesion from any angle by seeing through the overlying tissue.

The 3D printing process technique involves image analysis and segmentation strategies, followed by processing to preprint software in the form of a standard STL file format. Subsequently, additive manufacturing is employed via a Connex 500 (Stratasys) poly-jet printer producing prints, including up to 2 resins simultaneously, at a detail of 16 microns.

For the open surgical cases, sizing fidelity was assessed by comparing intraoperative photographs with model photographs taken from the same angle of approach (Figs. 3 and 4 and Video 1).

VIDEO 1. Intraoperative video clip demonstrating exposure of the feeding arterial vessels (some colored gray as a result of Onyx casting). The operative approach mirrored the simulated approach using the printed model. Copyright Edward Smith. Published with permission. Click here to view with Media Player. Click here to view with Quicktime.

Measurements of anatomical structures were made intraoperatively and compared with the same structures on 3D models (Fig. 5). From the collection of all 3 surgical models, a total of 5 structures were assessed, selected based on 2 factors: visibility in the surgical field (as not all lesional tissue was exposed in each case), and size of $2 \mathrm{~mm}$ or greater (to allow the use of a ruler with millimeter increments and also to corroborate with the use of a known 2-mm suction device as a reproducible photographic standard within visualized fields). Each measurement was repeated in triplicate and used to validate the accuracy of the model in comparison with actual surgical anatomy.

\section{Results \\ Model Fidelity}

Assessment of 5 areas in 3 surgical models demonstrated high fidelity between 3D models and actual operative anatomy, with $<10 \%$ deviation. Measurements on the model and in the operative field were each taken in triplicate and compared for deviation. Overall, there was $98 \%$ agreement ( $\mathrm{n}=5$, range $94 \%-100 \%)$ in measurements in vessel size, distance from AVM to ventricle, and distance from outer edge of nidus to feeding artery. A summary of these results is provided in Table 1, and a representative operative photograph is shown in Fig. 5.

\section{Clinical Results}

Successful embolization of the VOGM was accomplished without complication. Visual comparison of the right and left anterior cerebral arterial segments supplying the lesion suggested the more favorable avenue of approach for microcatheter navigation, based on vessel 

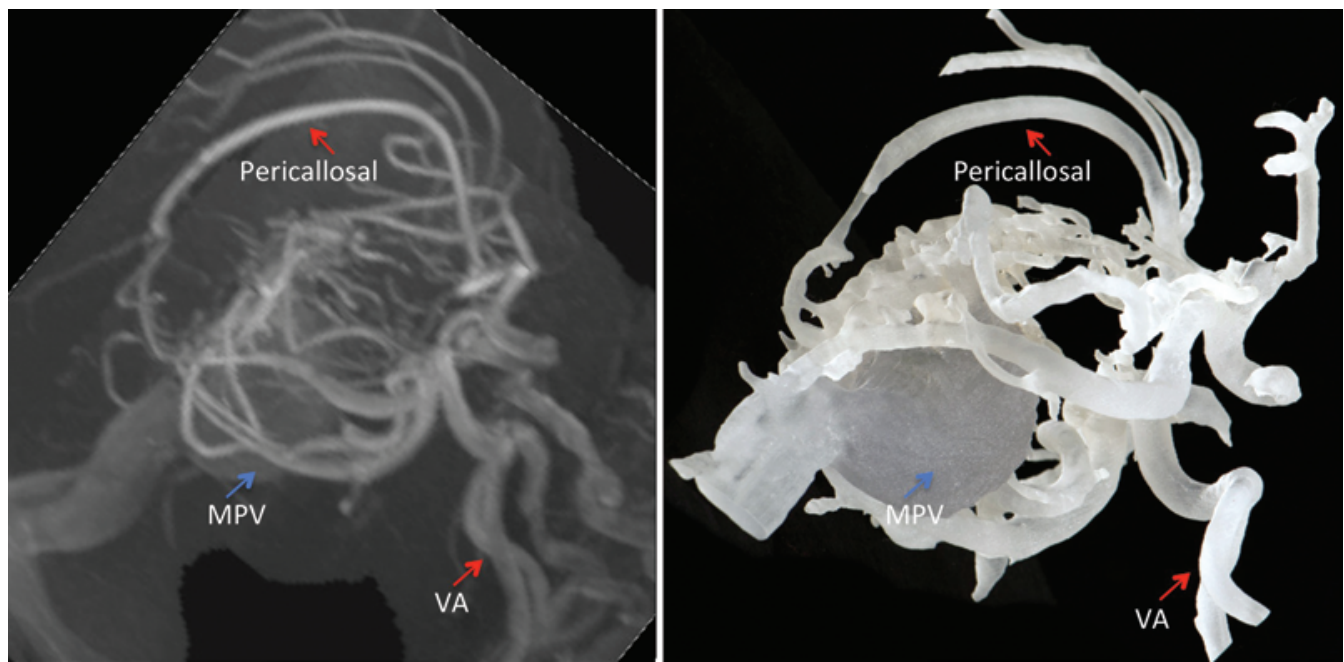

FIG. 3. Left: Maximum intensity projection MR angiogram obtained in a 2-month-old patient with a VOGM. Right: 3D print model of the same lesion. Red arrows designate arterial feeders from the pericallosal division of the anterior cerebral arteries toward the top of each image and the vertebral arteries (VA) toward the bottom. Blue arrows denote the median prosencephalic vein (MPV).

angulation. This assessment would have been extremely difficult to derive from $2 \mathrm{D}$ image displays, and the predicted ease of navigation was borne out during the embolization. Resection was accomplished in all 3 cases, with total angiographic obliteration confirmed by perioperative angiography (Fig. 6). No patient required transfusion and the only postoperative deficits included an expected transient hemiparesis and sensory loss in the large right parietal lesion, both of which had nearly completely resolved at 1 week postoperatively (independent ambulation, $4+/ 5$ strength at hospital discharge), with continued improvement. Operative time comparison was not available for the right parietal lesion, but using 2 cases of occipital AVMs with the same Spetzler-Martin grade, same location, and same patient age, both treated within 1 calendar year of the 3D model cases by the same surgeon as matched controls, the total surgical time (from initial incision to closure) was $12 \%$ faster in the 2 cases that used 3D models (on average, $\sim 30$ minutes faster with 3D models; nonmodel cases 285 and 288 minutes, 3D model cases 254 and 257 minutes).

\section{Discussion}

Treatment of complex neurovascular lesions such as AVMs and VOGMs requires careful application of meticulous surgical and/or endovascular technique. In par-

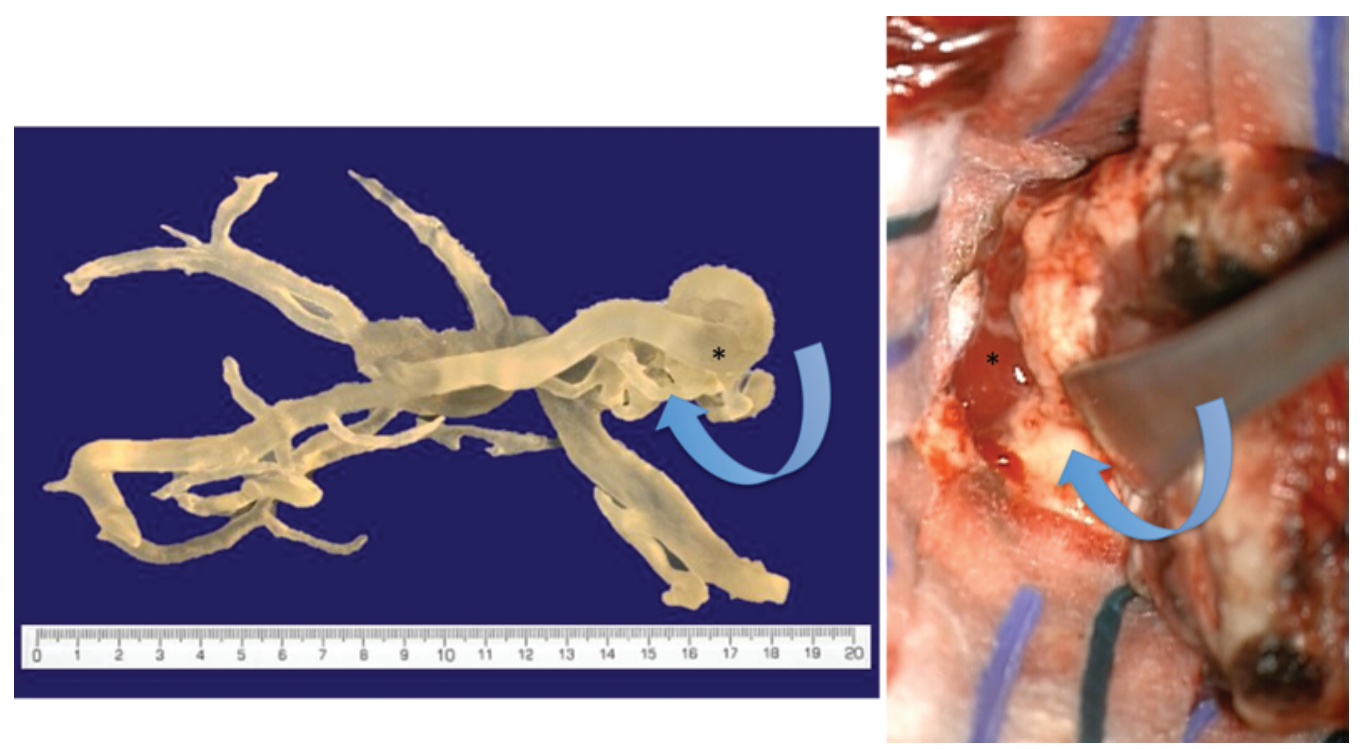

FIG. 4. Simulation of multiple surgical approaches using the 3D-printed models led to the decision to initially isolate the feeding arteries from an inferior-posterior approach, as denoted by the blue arrow in the model (left) and on an intraoperative photograph (right). (Asterisks denote deep draining vein.) The operative photograph reveals the vein, and the model predicted the arterial feeders to be located immediately medial. Preoperative surgical simulation aided in understanding relational anatomy for safe identification of the vessels. 


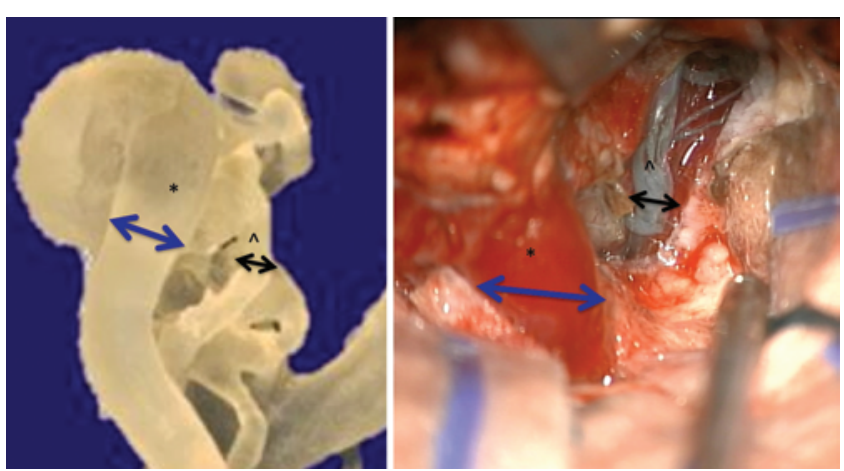

FIG. 5. Apposition of 3D-printed model (left) and operative photograph (right) demonstrating excellent concordance of predicted anatomy from model with actual visualization at operation. (Asterisks denote deep draining vein, carets identify feeding artery.) Double-ended arrowhead lines of same color represent identical lengths as measured in operative model and operative field.

ticular, risk is especially elevated in children, who have smaller blood volumes, complicated anesthetic requirements, and more difficult surgical and endovascular access as compared with adults. In other fields of medicine and surgery, the use of simulation has proven to be a useful tool to reduce therapeutic risk in complex medical conditions. ${ }^{1,4,7,8,10}$ In this proof-of-principle series, we report our initial experience with the novel application of $3 \mathrm{D}$ printing as a simulation tool translated directly to the operating room to improve the treatment of children with neurovascular disease.

The ability to employ multiple resins of varying consistency and color, coupled with submillimeter accuracy and production times measured in hours rather than weeks, has radically improved the verisimilitude of anatomical models that can potentially be delivered to clinicians overnight. ${ }^{5,8,9}$ This "on-demand" printing is, to our knowledge, a novel technique employed at our institution. Here we demonstrate that we can use standard clinical studiesMRI sequences taken as part of routine practice-and use these same images to produce high-fidelity 3D models tailor-made for each child. The only caveat is that the MRI sequences should include high-resolution 3D sequences that will allow for segmentation of brain parenchyma.

For delineation of arterial and venous anatomy, MR angiography and post-contrast T1-weighted volumetric sequences were used. Model production times do not include obtaining the pretreatment scan, but usually require 2-6 hours of imaging review and selection of model seg-

TABLE 1. Summary of average measurements of selected anatomical features of AVM cases

\begin{tabular}{lcc}
\hline & \multicolumn{2}{c}{ Average Measurement $(\mathrm{mm})$} \\
\cline { 2 - 3 } & Podel & Operative Field \\
\hline Vessel size $(n=12)$ & 3.5 & 3.7 \\
\hline $\begin{array}{l}\text { Edge of AVM nidus to lateral ventricle } \\
(n=6)\end{array}$ & 60 & 58 \\
\hline $\begin{array}{l}\text { Edge of AVM nidus to feeding artery } \\
(n=12)\end{array}$ & 54 & 57 \\
\hline
\end{tabular}

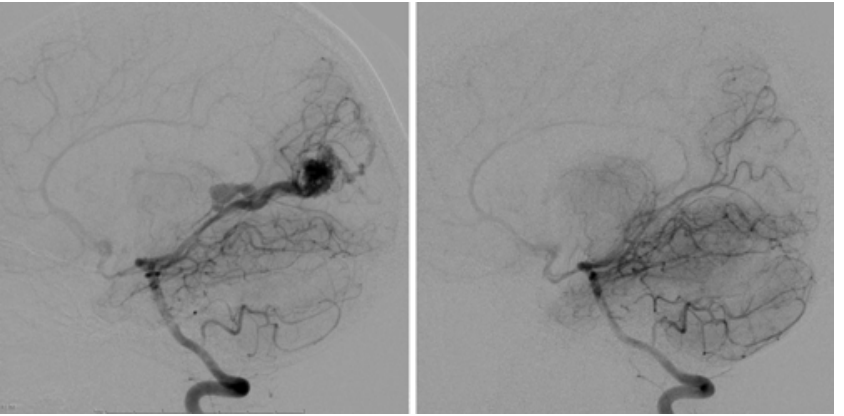

FIG. 6. Preoperative (left) and postoperative (right) lateral-projection catheter arteriograms obtained after vertebral artery injection, demonstrating complete resection of the AVM with preservation of normal vessels.

mentation, followed by 6-12 hours of actual printing and drying time (with variation dependent on the size of the model and the type of resins selected). Each scan can produce varying types of models for a given child: skeletonized vessels alone, a lesion in situ with a simulacrum of normal brain, or a colored lesion printed within transparent brain parenchyma. If desired, the lesion can be printed in an enlarged model, making details more obvious and easier to visualize in relation to other structures.

While readily acknowledging that the data presented here are limited due to the very small number of models, there is essential value in the provision of an illustrative, proof-of-principle result: the first study of 3D printing specific to pediatric AVM and VOGM treatment. We have demonstrated 3 important findings with this work. First, pediatric neurovascular lesions are printable in less than 24 hours using standard clinical imaging studies. Second, we have shown, for the first time to the best of our knowledge, direct correlation between 3D models and surgical anatomy with an objective, reproducible, high degree of fidelity (98\%). Finally, using closely matched controls, we can offer preliminary information that use of 3D printed models may potentially reduce operative time $(\sim 12 \%$ per case in 2 matched pairs of operations). This is only a feasibility study, given the small number of cases, but the findings suggest that further study into costs and benefits for institutions beyond improving training and surgical outcomes may be warranted with a larger cohort of patients.

In addition to our data showing manufacturing capability, realistic simulation of clinical anatomy, and potential improvements in therapeutic efficacy, we would remark that there was universal support for the use of 3D models as teaching tools by our group. Although the support is anecdotal in nature, both physicians and patients reported that handling the models led to greater understanding of the pathology and the implications of treatment. Independent of the objective evaluation of model fidelity and effects on treatment outcomes, these remarks suggest that additional study to better quantify the benefit of 3D models as teaching tools for students and family treatment discussions may be warranted.

\section{Conclusions}

With the appropriate team and expertise in place, printing 3D models of pediatric intracranial AVMs and 
VOGMs can be accomplished in less than 24 hours, using routine clinical MRI studies and recapitulating surgical and endovascular anatomy with extremely high fidelity; these prints may have direct benefit effects on the total perioperative pathway. Preliminary experience suggests that further study of 3D printing for cerebrovascular disease is warranted to explore its potential to improve therapeutic outcomes, reduce procedural times, and provide a novel method of physician and patient education.

\section{Acknowledgments}

We would like to acknowledge the support of the Lucas Warner AVM Research Fund and The Kids At Heart Neurosurgery Research Fund.

\section{References}

1. Abla AA, Lawton MT: Three-dimensional hollow intracranial aneurysm models and their potential role for teaching, simulation, and training. World Neurosurg 83:35-36, 2015

2. Akiba T, Inagaki T, Nakada T: Three-dimensional printing model of anomalous bronchi before surgery. Ann Thorac Cardiovasc Surg 20 Suppl:659-662, 2014

3. Costello JP, Olivieri LJ, Krieger A, Thabit O, Marshall MB, Yoo SJ, et al: Utilizing three-dimensional printing technology to assess the feasibility of high-fidelity synthetic ventricular septal defect models for simulation in medical education. World J Pediatr Congenit Heart Surg 5:421-426, 2014

4. Costello JP, Olivieri LJ, Su L, Krieger A, Alfares F, Thabit $\mathrm{O}$, et al: Incorporating three-dimensional printing into a simulation-based congenital heart disease and critical care training curriculum for resident physicians. Congenit Heart Dis 10:185-190, 2015

5. Rengier F, Mehndiratta A, von Tengg-Kobligk H, Zechmann $\mathrm{CM}$, Unterhinninghofen R, Kauczor HU, et al: 3D printing based on imaging data: review of medical applications. Int $\mathbf{J}$ CARS 5:335-341, 2010

6. Schmauss D, Haeberle S, Hagl C, Sodian R: Three-dimensional printing in cardiac surgery and interventional cardiology: a single-centre experience. Eur J Cardiothorac Surg 47:1044-1052, 2015

7. Schmauss D, Juchem G, Weber S, Gerber N, Hagl C, Sodian
R: Three-dimensional printing for perioperative planning of complex aortic arch surgery. Ann Thorac Surg 97:21602163, 2014

8. Silberstein JL, Maddox MM, Dorsey P, Feibus A, Thomas R, Lee BR: Physical models of renal malignancies using standard cross-sectional imaging and 3-dimensional printers: a pilot study. Urology 84:268-272, 2014

9. Watson RA: A low-cost surgical application of additive fabrication. J Surg Educ 71:14-17, 2014

10. Wurm G, Lehner M, Tomancok B, Kleiser R, Nussbaumer K: Cerebrovascular biomodeling for aneurysm surgery: simulation-based training by means of rapid prototyping technologies. Surg Innov 18:294-306, 2011

11. Zein NN, Hanouneh IA, Bishop PD, Samaan M, Eghtesad B, Quintini C, et al: Three-dimensional print of a liver for preoperative planning in living donor liver transplantation. Liver Transpl 19:1304-1310, 2013

\section{Author Contributions}

Conception and design: Smith, Weinstock, Prabhu, Orbach. Acquisition of data: all authors. Analysis and interpretation of data: Smith, Weinstock, Prabhu, Orbach. Drafting the article: Smith, Weinstock, Prabhu, Orbach. Critically revising the article: all authors. Reviewed submitted version of manuscript: all authors. Approved the final version of the manuscript on behalf of all authors: Smith. Administrative/technical/material support: Flynn.

\section{Supplemental Information Videos}

Video 1, Media Player. http://mfile.akamai.com/21490/wmv/ digitalwbc.download.akamai.com/21492/wm.digitalsource-naregional/peds14-677_video_1.asx.

Video 1, Quicktime. http://mfile.akamai.com/21488/mov/ digitalwbc.download.akamai.com/21492/qt.digitalsource-global/ peds14-677_video_1.mov.

\section{Correspondence}

Edward Smith, Department of Neurosurgery, Boston Children's Hospital and Harvard Medical School, 300 Longwood Ave., Boston, MA 02115. email: edward.smith@childrens.harvard.edu. 